\title{
ITERATED PATH INTEGRALS AND GENERALIZED PATHS ${ }^{1}$
}

\author{
BY KUO-TSAI CHEN
}

Communicated by Herbert Federer, June 15, 1967

Let $\mathfrak{M}$ be a $C^{\infty}$ manifold with a countable basis. For convenience, it is assumed that $\mathfrak{M}$ is Riemannian. Let $\mathfrak{B}$ be the set of "reduced" piecewise $C^{1}$ paths having a common initial point $p$ in $\mathfrak{M}$ such that each $\alpha \in \mathfrak{P}$ is parameterized by arc length. By a reduced path $\alpha:[0, l] \rightarrow \mathfrak{M}$, we mean one such that there exists no $t \in(0, l)$ with $\alpha(t-s)=\alpha(t+s)$ for $|s|$ sufficiently small.

Let $\Omega$ be the vector space (over the real number field $R$ ) of $C^{\infty}$ 1 -forms on $\mathfrak{M}$. Elements of $\Omega$ will be denoted by $w, w_{1}, w_{2}, \cdots$. Let $\alpha^{t}$ be the restriction $\alpha \mid[0, t], 0 \leqq t \leqq l$. Let $\int_{\alpha} w_{1}$ be the usual integral, and define, for $r>1$,

$$
\int_{\alpha} w_{1} \cdots w_{r}=\int_{0}^{l}\left(\int_{\alpha^{t}} w_{1} \cdots w_{r-1}\right) w_{r}(\alpha(t), \dot{\alpha}(t)) d t .
$$

Each iterated integral $\int w_{1} \cdots w_{r}$ is thus a real valued function on $\mathfrak{B}$. The totality of iterated integrals together with the constant functions on $\mathfrak{P}$ generates a subalgebra $F$ of the $R$-algebra of real valued functions on $\Re$. The $R$-algebra $F$ is of interest for two reasons: (a) Elements of $F$ have geometrical significance of the manifold $\mathfrak{M}$. (b) It follows from results in [1] that $F$ contains sufficiently many functions on $\mathfrak{P}$ as to separate the points of $\mathfrak{P}$.

The purpose of this note is to give some indication of the structure of $F$. In particular, Theorem 2 implies that, if $\mathfrak{M}=R^{n}$, then $F$ contains a dense subalgebra which is algebraically isomorphic with a polynomial algebra of, at most, countably many indeterminates.

We shall also introduce the notion of a generalized path in $\mathfrak{M}$ which is obtained through a process of dualization in a manner somewhat more complicated than that of a 1-dimensional current. (See [4].) The multiplication of generalized paths is nonabelian.

A detailed account will be given in a forthcoming paper.

1. Given any compact subset $K$ of $\mathfrak{M}$, define the seminorm \|\|$_{K}$ of $\Omega$ such that

${ }^{1}$ Work partially supported by the National Science Foundation under Grant NSF-GP-5423. 


$$
\|w\|_{K}=\sup \left\{\left\|w_{q}\right\|: q \in K\right\}
$$

where $\left\|w_{q}\right\|$ is the length of the cotangent vector $w_{q}$. Let $\operatorname{Tr}(\Omega)$ $=\Omega \otimes \cdots \otimes \Omega$ be the $r$-fold tensor product of $\Omega$ over $R$. If $u_{r} \in \operatorname{Tr}(\Omega)$, define $\left\|u_{r}\right\|_{K}$ to be the infimum of all $\sum_{i}\left\|w_{1}^{(i)}\right\|_{K} \cdots\left\|w_{r}^{(i)}\right\|_{K}$ for all possible finite sums $u_{r}=\sum_{i} w_{1}^{(i)} \otimes \cdots \otimes w_{r}^{(i)}$. Any element $u$ of the tensor algebra $T(\Omega)=\oplus_{r \geq 0} \operatorname{Tr}^{r}(\Omega)$ is a finite sum $u=\sum u_{r}, u_{r} \in T^{r}(\Omega)$, where $T^{0}(\Omega)=R$. Given a sequence $M=\left(M_{0}, M_{1}, \cdots\right)$ of positive numbers, define

$$
\|u\|_{M, K}=\sum M_{r}\left\|u_{r}\right\|_{K}
$$

where $\left\|u_{0}\right\|_{K}=\left|u_{0}\right|$. Then $T(\Omega)$ becomes a locally convex $R$-algebra whose topology is generated by all seminorms \|\|$_{M, K}$.

Define, in the tensor algebra $T(\Omega)$, a bilinear multiplication $\circ$ called the shuffle multiplication such that, for $r \geqq 0, s \geqq 0$, $\left(w_{1} \otimes \cdots \otimes w_{r}\right) \circ\left(w_{r+1} \otimes \cdots \otimes w_{r+8}\right)=\sum w_{\sigma(1)} \otimes \cdots \otimes w_{\sigma(r+8)}$ summing over those permutations $\sigma$ of the set $\{1, \cdots, r+s\}$ with $\sigma^{-1}(1)<\cdots<\sigma^{-1}(r), \sigma^{-1}(r+1)<\cdots<\sigma^{-1}(r+s)$. We shall assume that $w_{1}, \cdots, w_{r}=1$ for $r=0$. Then, under the shuffle multiplication, $T(\Omega)$ becomes a unitary commutative $R$-algebra $\operatorname{Sh}(\Omega)$. Since the shuffle multiplication is continuous, $\operatorname{Sh}(\Omega)$ is a locally convex $R$ algebra.

2. Define a linear map $j: T(\Omega) \rightarrow F$ such that $j 1=1$ and $j\left(w_{1} \otimes \cdots \otimes w_{r}\right)=\int w_{1} \cdots w_{r}$. Then $j$ is surjective. Moreover, for any $u, v \in T(\Omega)$,

$$
j(u \circ v)=j(u) j(v)
$$

so that $j$ is an epimorphism from the $R$-algebra $\operatorname{Sh}(\Omega)$ onto the $R$ algebra $F$. (This observation is essentially due to Ree [6].)

Given $\alpha \in \mathfrak{P}$, denote by $e_{\alpha}: F \rightarrow R$ the evaluation map at $\alpha$. Then

$$
e_{\alpha} j\left(w_{1} \otimes \cdots \otimes w_{r}\right)=\int_{\alpha} w_{1} \cdots w_{r} .
$$

It can be shown that $e_{\alpha} j: \operatorname{Sh}(\Omega) \rightarrow R$ is continuous. Then ker $e_{\alpha} j$ is a closed ideal of $\operatorname{Sh}(\Omega)$. Therefore $\operatorname{ker} j=\bigcap_{\alpha \in \mathfrak{B}}$ ker $e_{\alpha} j$ is a closed ideal of $\mathrm{Sh}(\Omega)$. We topologize the $R$-algebra $F$ through the isomorphism $\operatorname{Sh}(\Omega) / \operatorname{ker} j \cong F$.

3. If $f$ is a $C^{\infty}$ function on $\mathfrak{M}$ and if $\alpha \in \mathfrak{P}$, then 


$$
\begin{aligned}
\int_{\alpha} w_{1} & \cdots w_{r}(f w) w_{r+1} \cdots w_{r+s} \\
= & \int_{\alpha}\left(\left(w_{1} \cdots w_{r}\right) \circ d f\right) w_{r+1} \cdots w_{r+s} \\
& +f(p) \int_{\alpha} w_{1} \cdots w_{r} w w_{r+1} \cdots w_{r+8} .
\end{aligned}
$$

It follows that ker $j$ contains $I_{p}$ which is the closure of the subspace of $\mathrm{Sh}(\Omega)$ spanned by all elements of the type $u(f w) v-(u \circ d f) w v$

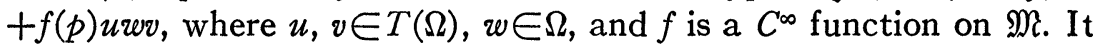
can be shown that $I_{p}$ is an ideal of $\operatorname{Sh}(\Omega)$. Consequently $j$ induces a continuous epimorphism $j_{p}: \operatorname{Sh}(\Omega) / I_{p} \rightarrow F$.

For any subspace $\Omega_{0}$ of $\Omega$, the inclusion $\Omega_{0} C \Omega$ induces a continuous homomorphism $\operatorname{Sh}\left(\Omega_{0}\right) \rightarrow \operatorname{Sh}(\Omega)$. If $\operatorname{dim} \Omega_{0}=1$, then $\operatorname{Sh}\left(\Omega_{0}\right)$ is isomorphic with the polynomial algebra $R(x)$; if $1<\operatorname{dim} \Omega_{0}<\infty$, it is known that $\operatorname{Sh}\left(\Omega_{0}\right) \cong R\left(x_{1}, x_{2}, \cdots\right)$.

Theorem 1. There exist $C^{\infty}$ functions $h_{1}, \cdots, h_{m}$ on $M$ with $\frac{1}{2}(m-1) \leqq \operatorname{dim} M$ such that, if $\Omega_{0}$ is the subspace of $\Omega$ spanned by $d h_{1}, \cdots, d h_{m}$, then $\mathrm{Sh}\left(\Omega_{0}\right)$ has a dense image in $\mathrm{Sh}(\Omega) / I_{p}$ under the composite homomorphism $\mathrm{Sh}\left(\Omega_{0}\right) \rightarrow \mathrm{Sh}(\Omega) \rightarrow \mathrm{Sh}(\Omega) / I_{p}$.

COROLlaRy. The algebra $F$ has a dense subalgebra which is a homomorphic image of a polynomial algebra with, at most, countably many indeterminates.

In the case of $\mathfrak{M}=R^{n}$, we have an additional result.

THEOREM 2. If $\Omega$ is the space of $C^{\infty} 1$-forms on $R^{n}$ and if $\Omega_{0}$ is the subspace spanned by the 1 -forms $d x^{1}, \cdots, d x^{n}$, where $x^{1}, \cdots, x^{n}$ are the coordinates of $R^{n}$, then the composite homomorphism

is injective.

$$
\mathrm{Sh}\left(\Omega_{0}\right) \rightarrow \operatorname{Sh}(\Omega) \stackrel{J}{\rightarrow} F
$$

4. Definition. A continuous homomorphism $\alpha$ : Sh $(\Omega) \rightarrow R$ such that $\alpha 1=1$ and $\alpha\left(I_{p}\right)=0$ is called a generalized path in $\mathfrak{M}$ with the initial point $p$.

The generalized path $\epsilon$ such that $\epsilon\left(T^{r}(\Omega)\right)=0$ for all $r \geqq 1$ is called the constant generalized path. Every generalized path $\alpha \neq \epsilon$ has a unique initial point $p$ and a unique terminal point $q$ such that, for any $C^{\infty}$ function $f$ on $\mathfrak{M}, \alpha(d f)=f(q)-f(p)$.

If $\alpha$ and $\beta$ are generalized paths in $\mathfrak{M}$, we define $\alpha \beta$ : Sh $(\Omega) \rightarrow R$ such that 


$$
\alpha \beta\left(w_{1} \otimes \cdots \otimes w_{r}\right)=\sum_{0 \leqq i \leqq r} \alpha\left(w_{1} \otimes \cdots \otimes w_{i}\right) \beta\left(w_{i+1} \otimes \cdots \otimes w_{r}\right) .
$$

THEOREM 3. If $\alpha$ and $\beta$ are generalized paths from $p$ to $q$ and from $q$ to $q^{\prime}$ respectively, then $\alpha \beta$ is a generalized path from $p$ to $q^{\prime}$. Moreover, there exists a generalized path $\alpha^{-1}$ from $q$ to $p$ such that $\alpha^{-1} \alpha=\alpha \alpha^{-1}=\epsilon$.

Obviously $\alpha \epsilon=\epsilon \alpha=\alpha$.

\section{BIBLIOGRAPHY}

1. K. T. Chen, Integration of paths, a faithful representation of paths by noncommutative formal power series, Trans. Amer. Math. Soc. 89 (1958), 395-407.

2. - Formal differential equations, Ann. of Math. 73 (1961), 110-133.

3. - Algebraization of iterated integration along paths, Bull. Amer. Math. Soc. 73 (1967), 975-978.

4. H. Federer and W. H. Fleming, Normal and integral currents, Ann. of Math. 72 (1960), 458-520.

5. H. H. Johnson, $A$ generalization of $K$. T. Chen's invariants for paths under transformation groups, Trans. Amer. Math. Soc. 105 (1962), 453-461.

6. R. Ree, Lie elements and an algebra associated with shuffles, Ann. of Math. 68 (1958), 210-220.

7. G. deRham, Variêtés differentiables, Hermann, Paris, 1955.

State University of New York at Buffalo and

UNIVERSITY OF ILLINOIS 